\title{
Application of fuzzy sets to structural reliability of existing structures
}

\author{
I. Mura \\ Department of Structural Engineering, University of Cagliari, \\ Cagliari, Italy
}

\begin{abstract}
Assessing the safety of a structure through inspection has always been part of the practice of the structural engineer. Information obtained through these inspections can be both objective and subjective. One can update the reliability of an existing structure based on objective and subjective information through the fuzzified Bayes theorem.

This paper cites the algorithm for computing the posterior probability of failure based on visual inspection of existing structures by incorporating fuzzy set theory into the Bayes theorem.

The failure design probability of a structural steel frame is updated.

Results indicate that the preciseness of the membership function associated with the input data has little or no influence on posterior failure probability. Keywords: reliability, Bayes theorem, fuzzy sets, existing structures, steel frame.
\end{abstract}

\section{Introduction}

In engineering practice one often has to deal with qualitative and vague evaluations, commonly known as "fuzzy". Such subjective fuzzy information is to be found in the results of inspections of structures (such as bridges, frames and so on) and are accompanied by those of a deterministic and probabilistic kind.

In particular, when we consider the question of evaluation of the safety level of an existing structure, it appears evident that we cannot avoid taking into account the quality of workmanship, the state of conservation of the elements making up the structure and so on. Estimation of these qualities can be expressed subjectively through variations of a linguistic nature. The values of these variables, as for example "the quality of the workmanship is good", or "the state 
of conservation of the bolts is poor", being vague and imprecise (which is to say fuzzy) cannot be defined with any certainty. For this reason, it is impossible to use them efficiently in the ambit of conventional statistical theories.

Modeling and the taking into consideration of vague and imprecise information can be performed through use of the fuzzy set theory, which was formulated by Zadeh in the 1960s and later developed by many other researchers (see Zadeh [1], Kaufmann and Gupta [2]). The first application of fuzzy set theory in civil engineering goes back to Blockley [3], who developed a method for evaluating the influence of the many parameters that may reduce the $a$ priori safety factor $n$ which determines the probability $\left(\mathrm{p}_{\mathrm{f}}=10^{-n}\right)$ of the occurrence of a given structural failure by means of linguistic variables and a "fuzzifier".

Subsequently, the procedure of taking into account subjective information was developed to allow the reaching of different goals. Blockley [4] extended the method illustrated in [3] to the study of twenty-three different structural failures. Brown and Yao [5] estimated the effective strength of cast concrete in a given structure starting from the results of compression tests on test pieces through the taking into account of a fuzzy parameter appropriate for defining the quality of the cast. Itoh and Itagaki [6] and Chou and Yuan [7] considered the problem of evaluating the reliability of existing structures. The algorithms they described allow the calculation of posterior probability based on the results of the visual inspection of structural components by incorporating the fuzzy set theory into the Bayes theorem. $\mathrm{Wu}[8]$ applied the Bayes theorem to evaluation of the reliability of systems whose identifying parameters are assumed as fuzzy random variables with an a priori distribution of the fuzzy kind.

Thus by combining the fuzzy set theory (which allows the expression of linguistic evaluations through specific functions called "membership functions") with the Bayes theorem it is possible to solve the problem of evaluating the reliability of an existing structure and determine the posterior reliability of uncertain parameters on the basis of all the results (deterministic, probabilistic and fuzzy) of inspections.

But the use of membership functions requires the solution of the problem of their modeling. The analysis and evaluation of damage to a structural element is in fact a difficult process in which human judgement plays a most important role. In the literature we can find many different methods proposed for the modeling of membership functions based on expert judgements. These operations are generally quite costly and laborious, as well as requiring long periods of time. Thus there has been a tendency to restrict membership functions to well-known forms. Triangular, left-shoulder, right-shoulder and trapezoidal or, more generally, piecewise linear, functions are common. Also used are standard Gaussian or Sigmoid type curves.

In this work we first illustrate the essential functions of Bayes' fuzzified theorem (Asai and Negoita [10], Kandel [11]). In the illustration, the theorem will be applied to the updating of design failure probability for the steel frame structure previously studied in [7], based on inspection results considered as fuzzy parameters. To evaluate the influence of the form of the membership functions necessary in defining inspection judgements on the final result, we use 
those proposed in [3] and [7] (triangular and piecewise linear in form) in our calculations. The two solutions obtained are then compared.

\section{The fuzzified Bayes theorem}

The Bayes theorem provides a method that allows inclusion of new information in a priori probabilistic evaluations, thus producing a new probability value for the occurrence of an event (Benjamin and Cornell [9]).

When there is no fuzzification and we take into account the theorem of total probability, the Bayes theorem is defined by the relation:

$$
P\left(B_{J} \mid A\right)=\frac{P\left(A \mid B_{J}\right) \cdot P\left(B_{J}\right)}{\sum_{k=1}^{m} P\left(A \mid B_{k}\right) \cdot P\left(B_{k}\right)}
$$

In Eqn (1) $B_{J}$ is the $J^{\text {th }}$ unknown random parameter having a known a priori distribution $P\left(B_{J}\right)$, whose probability is to be updated; $A$ is the random sample representing the parameter to be inspected; $m$ is the number of mutually exclusive and totally exhaustive events. $P\left(B_{J} \mid A\right)$ is commonly known as the posterior probability of $B_{J}$ after statistical event $A$ has taken place.

Equation (1) is thus valid if event $A$ can be expressed objectively, which is not the case of a fuzzy event. Therefore the above formulation of the Bayes theorem cannot be used with information of the type: "the crack is small" or "the state of bolt conservation is poor", and so on, which are vague and imprecise and cannot by defined with any certainty. To express such fuzzy information, specific linguistic variables are introduced. In particular, function $\mu_{\tilde{A}}(x)$ is introduced to express the probability of fuzzy event $\tilde{A}$.

Taking this into account, we find that the conditional probability of fuzzy event $\tilde{A}$ can be expressed as:

$$
P\left(\tilde{A} \mid B_{J}\right)=\int_{x} \mu_{\widetilde{A}}(x) \cdot f_{x \mid B_{J}}(x) \cdot d x
$$

where $f_{x \mid B_{J}}(x)$ is the conditional probability function that predicts event $B_{J}$ for support $x$. Equation (2) is valid if the support is continuous while if the support is discrete, the equation is written:

$$
P\left(\tilde{A} \mid B_{J}\right)=\sum_{\forall x} \mu_{\widetilde{A}}(x) \cdot P_{x \mid B_{J}}(x)
$$

Briefly stated, the posterior probability of event $B_{J}$, which takes into account the observation of fuzzy event $\tilde{A}$, is obtained by modifying Eqn (1) in accordance with Eqns (2) and (3).

The fuzzy Bayes theorem ([10], [11]) can therefore be expressed by the following relations. Where the support is continuous, taking into account Eqn (2), Eqn (1) is written: 


$$
P\left(B_{J} \mid \widetilde{A}\right)=\frac{\left[\int_{x} \mu_{\widetilde{A}}(x) \cdot f_{x \mid B_{J}}(x) \cdot d x\right] \cdot P\left(B_{J}\right)}{\sum_{k=1}^{m}\left[\int_{x} \mu_{\widetilde{A}}(x) \cdot f_{x \mid B_{k}}(x) \cdot d x\right] \cdot P\left(B_{k}\right)}
$$

where the support is discrete, taking into account Eqn (3), Eqn (1) is written:

$$
P\left(B_{J} \mid \widetilde{A}\right)=\frac{\left[\sum_{\forall x} \mu_{\widetilde{A}}(x) \cdot P_{x \mid B_{J}}\right] \cdot P\left(B_{J}\right)}{\sum_{k=1}^{m}\left[\sum_{\forall x} \mu_{\widetilde{A}}(x) \cdot P_{x \mid B_{k}}\right] \cdot P\left(B_{k}\right)}
$$

\section{Examples of application}

The example under consideration (see [7]) concerns the updating of the design failure probability of a steel frame following an inspection. It is assumed that such a probability is equal to $P_{f}=10^{-5}$, and that this represents a mean reliability value that could be calculated by considering the construction of a large number of similar structures. Numerous components (both structural and non-structural) are inspected. Since the conditions of these components influence the overall failure probability of the structure, they assume the role of probability parameters. It is supposed that during the inspection the following parameters are considered: connections, foundations, alignment, columns, beams, braces, bolts and paint.

\subsection{Statistical parameters}

We consider event $B_{J}$ which may correspond to event $B_{1}$ (= the structural component is safe) or to event $B_{2}$ (= the structural component is near failure).

The conditional probability function of random event $B_{J}$, owing to the support of quality $x$, supposedly assumes the following quadratic forms:

$$
\begin{gathered}
g\left(X \mid B_{1}\right)=\left(\frac{N_{i}-6}{6}\right)(0.1)^{2}(x-10)^{2}+1.0 \\
g\left(X \mid B_{2}\right)=\left(\frac{N_{i}-6}{6}\right)(0.1)^{2} \cdot x^{2}+1.0
\end{gathered}
$$

since $N_{i}$ is the index of the importance of the parameter or structural component $i$ and $x$ is the support quality. Since the above functions (6) represent probability functions, they must satisfy the basic axiom of probability. They are therefore normalized in accordance with the following relation:

$$
P_{i}\left(X \mid B_{J}\right)=\frac{g\left(x \mid B_{J}\right)}{\sum_{\forall x} g\left(x \mid B_{J}\right)}
$$




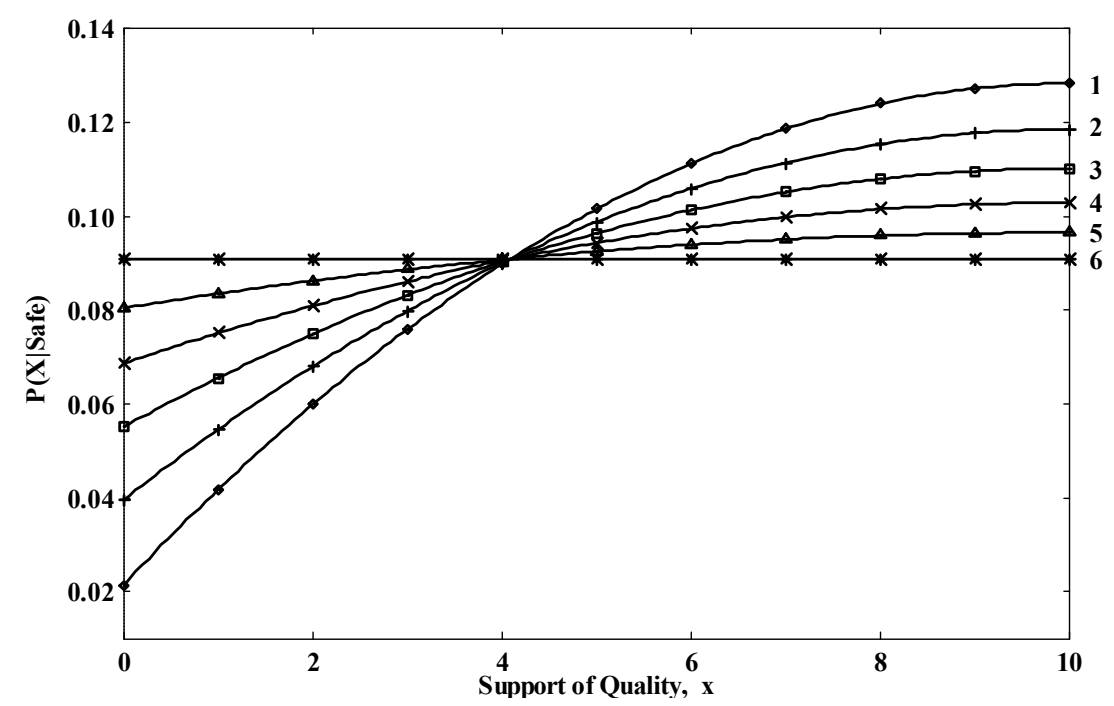

Figure 1: Conditional probability function for Quality at various importance indices given as Safe.

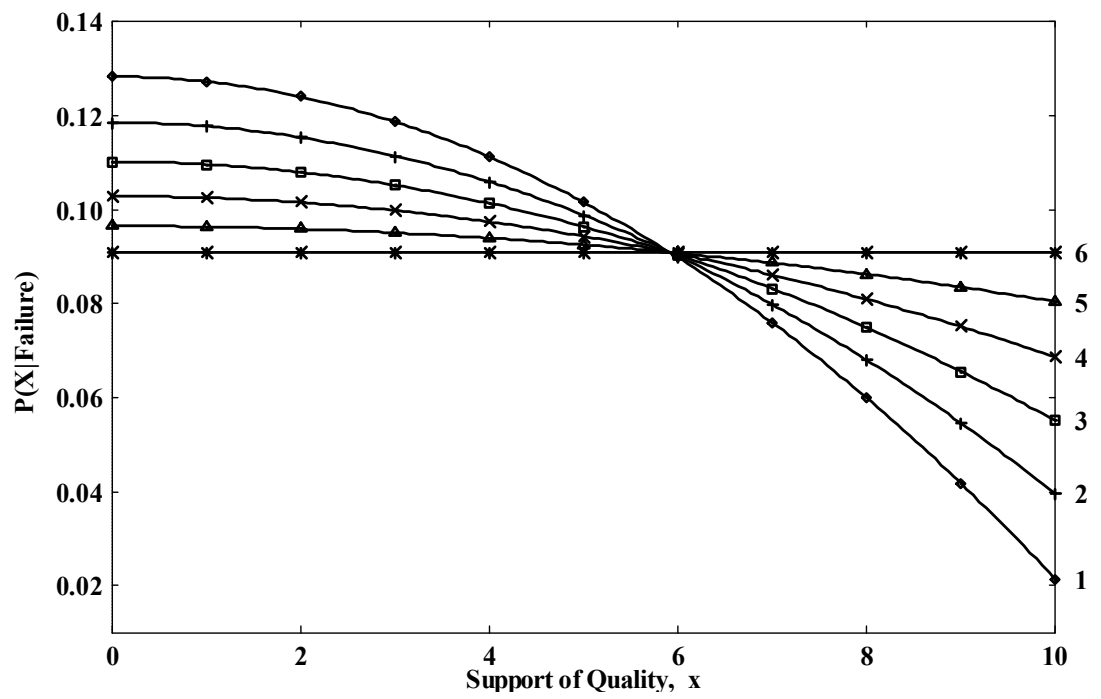

Figure 2: Conditional probability function for Quality at various importance indices given as Failure.

Functions (6) must be defined so as to respect the "weight" assumed by each parameter in the evaluation of the overall safety of the structure. The meaningfulness of each parameter in overall safety is given by the indices of 
importance. Parameters are ordered on the basis of their indices of importance. Indices of unit value correspond to the maximum importance. Numerically increasing values of the index imply a reduction in the importance of parameters.

In the part that follows it is supposed possible to assume for each index, on the basis of expert judgement, the values given in Table I. It is to be pointed out that functions (6) may assume different forms for each parameter. In particular, the index of importance may in such forms be defined implicitly.

The results of normalization calculations are shown in Figures 1 and 2.

Finally, in order to simplify calculations, we allow the parameters to be mutually independent, as are their effects on structural reliability.

\subsection{Probability of inspection results}

It is supposed that the quality of each of the parameters considered above can be verbally described by fuzzy evaluations of the kind: "the state of conservation of the bolts is poor". Below, in order to evaluate the influence of the modeling on the results supplied by the fuzzified theorem, two different circumstances will be considered. The first kind of modeling, which will be referred to as type 1 and which is taken from [3], is indicated in Figure 3 and corresponds to functions (8).

$$
\begin{aligned}
& \mu_{P}(x)=\left\{\begin{array}{llr}
-0.10 \cdot x+1.0 & \text { if } & x \leq 1 \\
-0.40 \cdot x+1.3 & \text { if } & 1 \leq x \leq 2 \\
-0.30 \cdot x+1.1 & \text { if } & 2 \leq x \leq 3 \\
-0.10 \cdot x+0.5 & \text { if } & 3 \leq x \leq 5 \\
0 & \text { if } & x \geq 5
\end{array}\right. \\
& \mu_{N}(x)=\left\{\begin{array}{llr}
0 & \text { if } & 0 \leq x \leq 2 \\
0.20 \cdot x-0.4 & \text { if } & 2 \leq x \leq 3 \\
0.40 \cdot x-1.0 & \text { if } & 3 \leq x \leq 5 \\
-0.40 \cdot x+3.0 & \text { if } & 5 \leq x \leq 7 \\
-0.20 \cdot x+1.6 & \text { if } & 7 \leq x \leq 8 \\
0 & \text { if } & 8 \leq x \leq 10
\end{array}\right. \\
& \mu_{G}(x)=\left\{\begin{array}{llr}
0 & \text { if } & x \leq 5 \\
0.10 \cdot x-0.5 & \text { if } & 5 \leq x \leq 7 \\
0.30 \cdot x-1.9 & \text { if } & 7 \leq x \leq 8 \\
0.40 \cdot x-1.7 & \text { if } & 8 \leq x \leq 9 \\
0.10 \cdot x & \text { if } & 9 \leq x \leq 10
\end{array}\right.
\end{aligned}
$$

The second kind of function modeling, which will be referred to as type 2, is taken from [7]; it is expressed by means of relations (9) and is indicated in Figure 4. 
High Performance Structures and Materials III 701

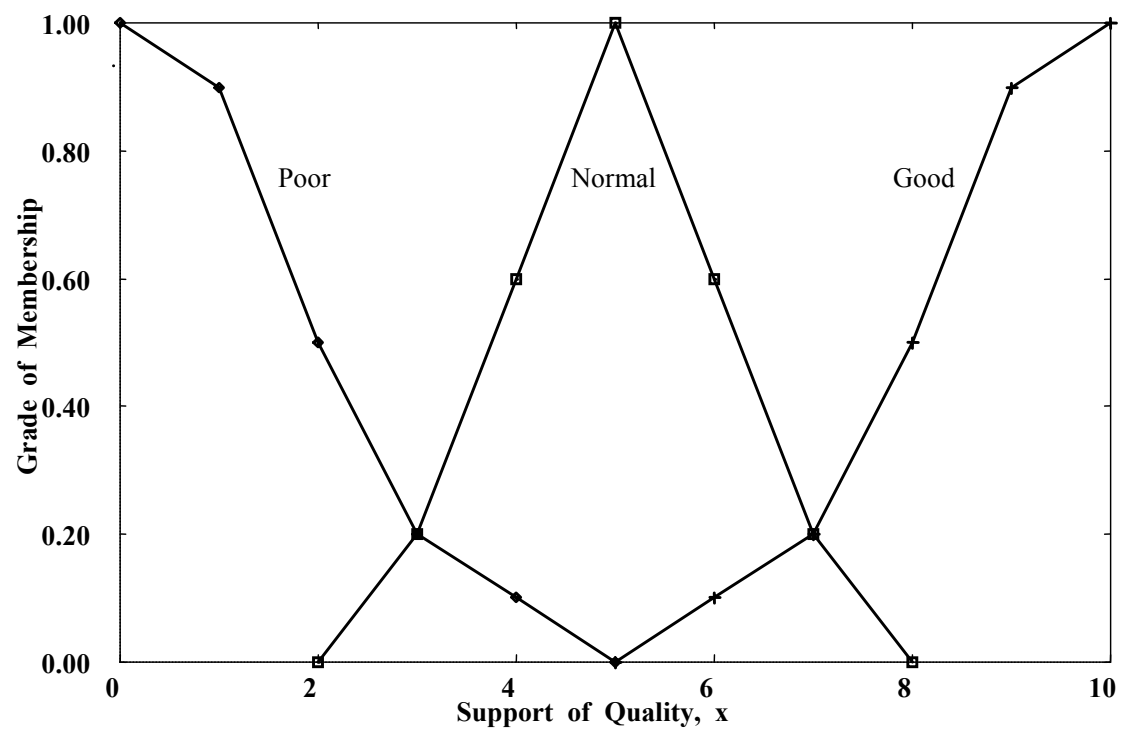

Figure 3: $\quad$ Membership function for Quality of type 1.

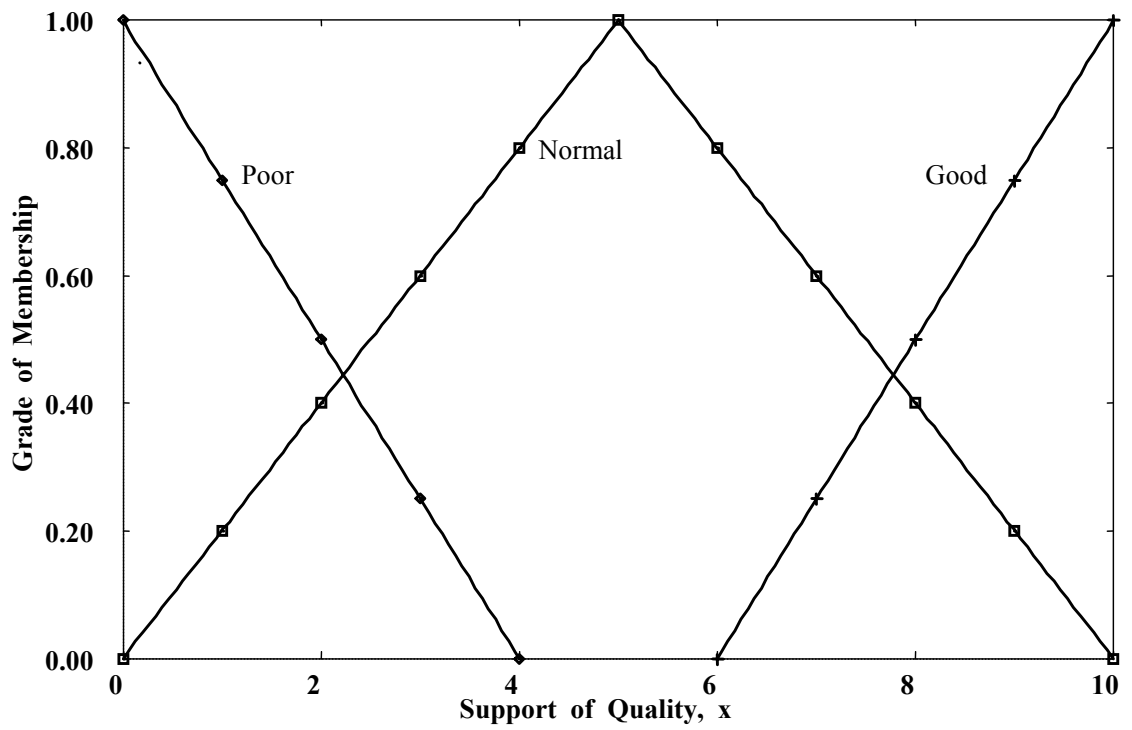

Figure 4: $\quad$ Membership function for Quality of type 2. 
702 High Performance Structures and Materials III

$$
\begin{aligned}
& \mu_{P}(x)= \begin{cases}-2.25 \cdot x+1.0 & \text { if } x \leq 4 \\
0 & \text { if } x>4\end{cases} \\
& \mu_{N}(x)=\left\{\begin{array}{lll}
0.20 \cdot x & \text { if } x \leq 5 \\
-0.20 \cdot x+2.0 & \text { if } x>5
\end{array}\right. \\
& \mu_{G}(x)=\left\{\begin{array}{lll}
0.25 \cdot x-1.5 & \text { if } x \leq 6 \\
0 & \text { if } x>6
\end{array}\right.
\end{aligned}
$$

Considering that three quality judgements can be applied to the eight parameters, 6561 possible combinations of inspection results can be considered. The three possible cases of inspection results that are considered herein (taken from [7]) are illustrated in Table I below.

\subsection{Updating of failure probability}

Having assigned the index of importance to each of the structural parameters considered, we can calculate the posterior failure probability of the frame both on the basis of functions (7) and the membership functions reflecting inspection results (8) and (9). The posterior probability of failure of the frame, taking into account the occurrence of fuzzy events $\tilde{A}_{1}, \tilde{A}_{2} \ldots \tilde{A}_{8}$, is obtained by extending Eqn (1) to the case of more than one fuzzy variable. We have:

$$
P\left(B_{J} \mid \widetilde{A}_{1}, \widetilde{A}_{2} \ldots . \widetilde{A}_{8}\right)=\frac{P\left(\widetilde{A}_{1}, \widetilde{A}_{2} \ldots \widetilde{A}_{8} \mid B_{J}\right) \cdot P\left(B_{J}\right)}{\sum_{k=1}^{2} P\left(\widetilde{A}_{1}, \widetilde{A}_{2} \ldots \tilde{A}_{8} \mid B_{k}\right) \cdot P\left(B_{k}\right)}
$$

Having supposed that events $\tilde{A_{1}}, \tilde{A_{2}} \ldots \tilde{A_{8}}$ are independent, we indicate by:

$$
P\left(B_{J}^{1}\right)=\frac{P\left(\tilde{A}_{1} \mid B_{J}\right) \cdot P\left(B_{J}\right)}{\sum_{k=1}^{2} P\left(\tilde{A}_{1} \mid B_{k}\right) \cdot P\left(B_{k}\right)}
$$

the posterior probability of event $B_{J}$ on the basis of observation of the first parameter. Obviously we also have the following:

$$
P\left(B_{J}^{2}\right)=\frac{P\left(\widetilde{A}_{2} \mid B_{J}\right) \cdot P\left(B_{J}^{1}\right)}{\sum_{k=1}^{2} P\left(\tilde{A}_{2} \mid B_{k}\right) \cdot P\left(B_{k}\right)}
$$

Having indicated by $P\left(B_{J}^{i}\right)$ the posterior probability of event $B_{J}$ following observation of parameter $i$, in general we can write: 


$$
P\left(B_{J}^{i}\right)=\frac{P\left(\tilde{A}_{i} \mid B_{J}\right) \cdot P\left(B_{J}^{i-1}\right)}{\sum_{k=1}^{2} P\left(\widetilde{A}_{i} \mid B_{k}\right) \cdot P\left(B_{k}^{i-1}\right)}
$$

Equation (13) above is immediately explicable by using Eqn (2) when the support is continuous and Eqn (3) when it is discrete.

Table 1: $\quad$ Posterior failure probability for the type 1 (col. 5) and type 2 (col. 6) membership functions adopted.

\begin{tabular}{|c|c|c|c|c|c|}
\hline $\begin{array}{c}\text { CASE N }^{\circ} \\
(1)\end{array}$ & $\begin{array}{l}\text { Parameter } \\
\text { (2) }\end{array}$ & $\begin{array}{c}\operatorname{Index~}^{\mathrm{N}_{i}} \\
\text { (3) }\end{array}$ & $\begin{array}{c}\text { Quality } \mathrm{A}_{i} \\
(4)\end{array}$ & $\begin{array}{c}\mathrm{P}_{f}^{i}(1) \\
(5)\end{array}$ & $\begin{array}{c}\mathrm{P}_{f}^{i}(2) \\
(6)\end{array}$ \\
\hline \multirow{8}{*}{1} & Connection & 1 & Normal & $1.00 \mathrm{E}-5$ & $1.00 \mathrm{E}-5$ \\
\hline & Foundation & 1 & Poor & $3.16 \mathrm{E}-5$ & $3.11 \mathrm{E}-5$ \\
\hline & Alignment & 1 & Normal & $3.16 \mathrm{E}-5$ & $3.11 \mathrm{E}-5$ \\
\hline & Columns & 2 & Poor & $6.94 \mathrm{E}-5$ & $6.76 \mathrm{E}-5$ \\
\hline & Beams & 4 & Normal & $6.94 \mathrm{E}-5$ & $6.76 \mathrm{E}-5$ \\
\hline & Bracing & 4 & Poor & $9.52 \mathrm{E}-5$ & $9.24 \mathrm{E}-5$ \\
\hline & Bolts & 4 & Poor & $1.31 \mathrm{E}-4$ & $1.26 \mathrm{E}-5$ \\
\hline & Painting & 6 & Normal & $1.31 \mathrm{E}-4$ & $1.26 \mathrm{E}-5$ \\
\hline \multirow{8}{*}{2} & Connection & 1 & Good & $3.17 \mathrm{E}-6$ & $3.22 \mathrm{E}-6$ \\
\hline & Foundation & 1 & Normal & $3.17 \mathrm{E}-6$ & $3.22 \mathrm{E}-6$ \\
\hline & Alignment & 1 & Poor & $1.00 \mathrm{E}-5$ & $1.00 \mathrm{E}-5$ \\
\hline & Columns & 2 & Normal & $1.00 \mathrm{E}-5$ & $1.00 \mathrm{E}-5$ \\
\hline & Beams & 4 & Good & $7.29 \mathrm{E}-6$ & $7.32 \mathrm{E}-6$ \\
\hline & Bracing & 4 & Normal & $7.29 \mathrm{E}-6$ & 7.32E-6 \\
\hline & Bolts & 4 & Poor & $1.00 \mathrm{E}-5$ & $1.00 \mathrm{E}-5$ \\
\hline & Painting & 6 & Good & $1.00 \mathrm{E}-5$ & $1.00 \mathrm{E}-5$ \\
\hline \multirow{8}{*}{3} & Connection & 1 & Good & $3.17 \mathrm{E}-6$ & $3.22 \mathrm{E}-6$ \\
\hline & Foundation & 1 & Good & $1.00 \mathrm{E}-6$ & $1.04 \mathrm{E}-6$ \\
\hline & Alignment & 1 & Good & $3.17 \mathrm{E}-7$ & $3.34 \mathrm{E}-7$ \\
\hline & Columns & 2 & Normal & $3.17 \mathrm{E}-7$ & $3.34 \mathrm{E}-7$ \\
\hline & Beams & 4 & Good & $2.32 \mathrm{E}-7$ & $2.44 \mathrm{E}-7$ \\
\hline & Bracing & 4 & Normal & $2.32 \mathrm{E}-7$ & $2.44 \mathrm{E}-7$ \\
\hline & Bolts & 4 & Good & $1.69 \mathrm{E}-7$ & $1.79 \mathrm{E}-6$ \\
\hline & Painting & 6 & Normal & $1.69 \mathrm{E}-7$ & $1.79 \mathrm{E}-6$ \\
\hline
\end{tabular}

\section{Results of numerical processing and conclusions}

Results of calculations performed are shown in Table 1 and correspond to the three circumstances of the inspection results considered.

In column 5 of Table 1 we find the updated values of failure probability deduced by adopting functions (8); in column (6) we see those deduced by adopting functions (9). 
Calculations were carried out by adopting the hypothesis of a discrete support by means of the use of a simple program created specifically for the purpose.

The values of failure probabilities, later updated by means of Eqn (13), on the basis of inspection results, are found to be practically insensitive to the different definitions of the membership functions adopted and, to all practical purposes, substantially coincident.

As can easily be seen, an "average" failure probability, like that of the design, is reduced if following inspection it is found that the parameters that globally define it turn out to be more than average. On the contrary, it increases when it is found that the parameters that globally define it are less than average.

Generally speaking, the results indicate that the preciseness of the membership function associated with the input data has little or no influence on the posterior failure probability. Thus, the fuzzified Bayes theorem has practical applications in assessing the posterior failure probability of existing structures, with minimal expert input to establish the necessary membership functions and is a very powerful tool of analysis.

\section{References}

[1] Zadeh L.A., Fuzzy sets. Information and Control, Vol. 8, pp. 338-353, 1965.

[2] Kaufmann A., Gupta M.M., Fuzzy mathematical models in engineering and management science. North-Holland, Amsterdam, 1988.

[3] Blockley D.I., Predicting the likelihood of structural accidents. Proc. Inst Civ. Eng., 59(2), pp. 659-668, 1975.

[4] Blockley D.I., Analysis of structural failures. Proc. Inst Civ. Eng., 62(1), pp. 51-74, 1977.

[5] Brown C.B., Yao T.P., Fuzzy sets and structural engineering. Journal of Structural Engineering, ASCE, 109(5), pp. 1211-1225, 1983.

[6] Itoh S., Itagaki H., Application of Fuzzy-Bayesian Analysis to Structural Reliability. Proc. of ICOSSAR '89, ASCE, N.Y., pp. 1171-1174, 1989.

[7] Chou K.C., Yuan J., Fuzzy-Bayesian approach to reliability of existing structures. Journal of Structural Engineering, ASCE, 119(11), pp. 3276$3290,1993$.

[8] Wu H.C., Bayesian system reliability assessment under fuzzy environments. Reliability Engineering \& System Safety, 83, pp. 227-286, 2003.

[9] Benjamin J.R., Cornell C.A., Probability, statistics and decision for civil engineers. Mc Graw Hill, New York, N.Y, 1970.

[10] Asai K., Negoita C.V., Introduction to fuzzy set theory. Ohm Press, Tokio, 1978.

[11] Kandel A., Fuzzy mathematical techniques with applications. Addison Wesley, Reading, Massachusset, 1986. 